\title{
Mass Selective Axial Ion Ejection from Linear Quadrupoles with Added Octopole Fields
}

\author{
Annie Moradian and D. J. Douglas \\ Department of Chemistry, University of British Columbia, Vancouver, British Columbia, Canada
}

\begin{abstract}
Mass selective axial ejection of ions from linear quadrupoles with added octopole fields is described. Quadrupoles with $2.0 \%$ and $2.6 \%$ added octopole fields have been tested and compared with a conventional quadrupole. The effects of trapping ions at different $q$ values, excitation voltage, scan direction, balanced and unbalanced $\mathrm{rf}$ voltages on the rods, and dc applied between the rods have been investigated. The highest scan speeds and best resolution are obtained with resonant excitation and ejection at high $q(q=0.8)$. With axial ejection, the quadrupole with a $2.0 \%$ added octopole field provides mass resolution and ejection efficiencies similar to a conventional rod set. Quadrupole, dipole, and simultaneous dipole-dipole excitation between the $x$ and $y$ rod pairs were compared, and no advantage was found with quadrupole or dipole-dipole excitation. The effects of scan speed were investigated and a resolution at half height of about 1600 is possible at scans speed up to $5000 \mathrm{Th} / \mathrm{s}$. (J Am Soc Mass Spectrom 2008, 19, 270-280) @ 2008 American Society for Mass Spectrometry
\end{abstract}

$\mathrm{L}$ inear quadrupole ion traps (LIT) have been known since the introduction of the quadrupole storage Jing in 1969 [1]. If a linear quadrupole is operated with radio frequency (rf) only voltages between the rod pairs, and if stopping potentials are applied to electrodes at the ends of the quadrupole, ions of a broad range of mass to charge ratios $(\mathrm{m} / \mathrm{z})$ can be trapped in the quadrupole. Linear traps have several advantages compared with 3-D traps [2]. Because there is no quadrupole field along the central axis, linear traps have higher injection efficiencies than 3-D traps. The larger volume of the linear trap enables storage of a greater number of ions without space charge effects.

In 1999, Hager [3] reported a new method of mass scanning with linear quadrupoles, called mass selective axial ejection (MSAE). The method uses the fringing field at the exit of the quadrupole. In the fringing field the otherwise independent ion motions in $\mathrm{x}, \mathrm{y}$, and $\mathrm{z}$ are coupled. The quadrupole is operated in rf-only mode with ions flowing continuously into the quadrupole. The end plate or exit lens is set at a stopping potential relative to the quadrupole rod offset. Within the fringing field, radial energy of the ions is converted into axial energy and ions can surmount the exit lens barrier to reach a detector. Ions with larger radial displacements in the fringing field receive greater axial kinetic energy increases than ions with smaller radial displacements. Thus, some form of radial excitation of ions is required for ion ejection.

The same concept was then used for axial ejection of ions trapped in a linear quadrupole [4]. Experiments were done with a triple quadrupole mass spectrometer.

Address reprint requests to Dr. D. J. Douglas, Department of Chemistry, University of British Columbia, 2036 Main Mall, Vancouver, BC, V6T 1Z1, Canada. E-mail: douglas@chem.ubc.ca
Fragment ions produced in tandem mass spectrometry were trapped in the collision cell $(\mathrm{Q} 2)$ or the last mass analyzing quadrupole (Q3), by applying stopping potentials to lenses at the ends of the quadrupoles. For axial ejection, ions were excited at their resonant frequencies by applying fixed-frequency auxiliary ac voltages to two or all four rods of a quadrupole or to the exit lens. By scanning the trapping rf amplitude, ions of different $\mathrm{m} / \mathrm{z}$ ratios came into resonance and were ejected to produce a mass spectrum.

The mass resolution (mass resolving power) at half height $\left(R_{1 / 2}\right)$ with axial ejection from Q2 $\left(4 \times 10^{-4}\right.$ torr $)$ was typically about 1000 at $\mathrm{m} / \mathrm{z} 609$ with a scan rate of $1000 \mathrm{Th} / \mathrm{s}$. Slower scans, $5 \mathrm{Th} / \mathrm{s}$, resulted in much higher resolution, $\boldsymbol{R}_{1 / 2}=6000$ at $\mathrm{m} / z$ 609. It was found that, because of the lower pressure in Q3 $\left(3 \times 10^{-5}\right.$ torr), $\mathrm{R}_{1 / 2}=6000$ could be obtained at the higher scan speed of $100 \mathrm{Th} / \mathrm{s}$. Experiments with fragment ions formed in Q2 and then trapped and axially ejected from Q3 showed 16 times greater sensitivity than a conventional triple quadrupole MS/MS scan [4].

It has been argued that for 3-D ion traps, it is advantageous to deliberately add higher field harmonics (octopole or hexapole) to the quadrupole field, because this increases the ion fragmentation efficiency and potentially gives higher resolution in ion ejection or isolation [5]. Addition of a positive even-order higher multipole to the field in the $\mathrm{z}$ direction of a 3-D trap introduces nonlinear effects; the most important is a shift of the frequency of ion oscillation with the amplitude of oscillation [6].

Douglas and coworkers $[7,8]$ have shown that an octopole field can be added to a linear quadrupole by making one pair of rods greater in diameter than the other pair. Experiments where trapped ions were reso- 
nantly excited to induce fragmentation showed that a rod set with $4 \%$ added octopole field can give higher efficiency in dissociating ions than a conventional rod set [8], especially at pressures of $1 \times 10^{-4}$ torr or less. It has been demonstrated that conventional mass analysis with applied dc and rf fields is possible with quadrupoles with $2.0 \%$ and $2.6 \%$ added octopole fields despite the large field distortions, provided the dc is applied with the correct polarity [9].

The higher fragmentation efficiency with rod sets with added octopole fields makes them attractive as ion traps in instruments where Q3 of a triple quadrupole must be used to fragment ions at low-pressure. However it has not yet been demonstrated that mass selective axial ejection is possible with such rod sets. Here we investigate axial ejection as a means of mass analysis of ions in a linear quadrupole with an added octopole field. We compare axial ejection with quadrupoles with $2.0 \%$ and $2.6 \%$ added octopole fields to axial ejection with a conventional round-rod linear quadrupole. The effects of trapping ions at different $q$ values, excitation voltage, scan direction, balanced and unbalanced rf voltage on the rods, and dc applied between the rods have been investigated. The highest scan speeds and best resolution are obtained with excitation and ejection at high $q$ (0.8). Quadrupole, dipole, and simultaneous dipole-dipole excitation between the $x$ and $y$ rods are compared and no advantage is found with quadrupole or dipole-dipole excitation. The results show MSAE of ions from linear quadrupoles with added octopole fields can give mass resolution comparable to that of a conventional rod set. The effects of scan speed were investigated and a resolution at half height of about 1600 is possible at scan speeds up to $5000 \mathrm{Th} / \mathrm{s}$ with a quadrupole with a $2.0 \%$ added octopole field.

\section{Experimental}

\section{Instrument}

The instrument used here is a single quadrupole mass spectrometer, shown in Figure 1a. Ions formed by pneumatically assisted electrospray ionization (ESI) pass through a $1.5 \mathrm{~mm}$ diameter aperture in a curtain plate, through a dry $\mathrm{N}_{2}$ curtain gas, and enter the vacuum system through a $0.0875 \mathrm{~mm}$ diameter orifice in the tip of a cone. Ions then travel through a quadrupole ion guide, Q0 $\left(8 \times 10^{-3}\right.$ torr of $\left.\mathrm{N}_{2}\right)$, where they are collisionally cooled. The $20 \mathrm{~cm}$ long Q0 rods are capacitively coupled to the Q1 drive rf voltage $(1.20 \mathrm{MHz})$. The linear ion trap, Q1, is separated from the ion guide by a lens with a $3.0 \mathrm{~mm}$ diameter aperture (the "entrance" lens), and is in a chamber pumped to $1.6 \times 10^{-5}$ torr. Ions leave Q1 and pass through two additional lenses, the first with a mesh covered $9 \mathrm{~mm}$ aperture, and the second with an open $9 \mathrm{~mm}$ aperture. The mesh-covered lens is referred to as the exit lens and is located $3.5 \mathrm{~mm}$ from the end of the quadrupole rod set. The second lens is spaced $5 \mathrm{~mm}$ from the exit lens. Ions

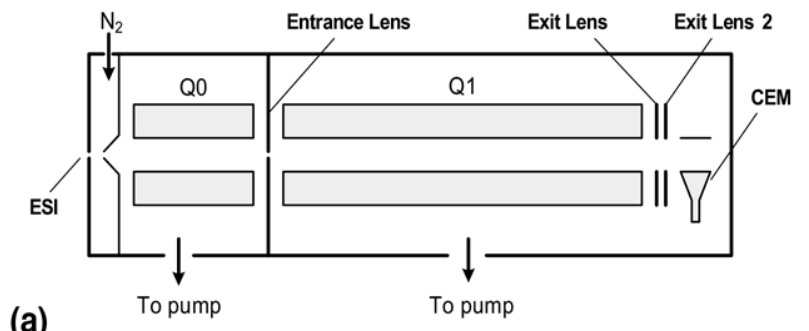

(a)

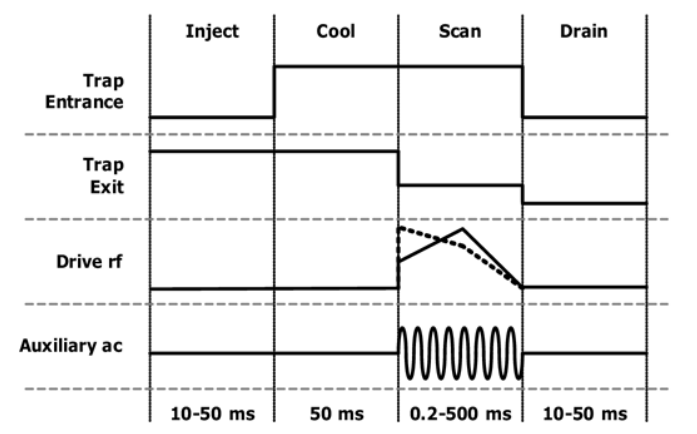

(b)

Figure 1. (a) Schematic of the linear ion trap system. ESI, electrospray ionization; Q0, rf only quadrupole ion guide; Q1, linear quadrupole trap; CEM, channel electron multiplier; (b) schematic of the timing parameters.

are detected via a continuous dynode electron multiplier CEM (Channeltron; Burle Electro-Optics, Sturbridge, MA), operated in ion counting mode.

Auxiliary ac from an arbitrary waveform generator (SRS DS345; Stanford Instruments, Sunnyvale, CA) was applied between one pair of rods $(x$ or $y)$ as dipole excitation or simultaneously between two pairs of rods as dipole-dipole excitation, or to all four rods as quadrupole excitation. Dipole excitation was applied through toroidal transformers [10]. When the same frequency was applied to both the $x$ and $y$ rods for dipole-dipole excitation, the waveforms were phase locked. When different frequencies were used, the waveforms were not phase locked. Quadrupole excitation was applied through the quadrupole power supply through a circuit supplied by MDSSCIEX. In a pure quadrupole field the angular frequencies of ion oscillation are given by

$$
\omega_{u}=\left(n+\frac{\beta_{u}}{2}\right) \Omega
$$

where $n=0, \pm 1, \pm 2, \ldots, u=x$ or $y, \beta$ is a function of the trapping voltage, and $\Omega$ is the angular frequency of the quadrupole rf voltage. Resonance occurs when the frequency $\omega_{e x}$ of the auxiliary voltage coincides with one of the free oscillation frequencies of ions $\omega_{u}$. With dipole excitation the fundamental frequencies $(n=0)$ were excited. The resonant frequencies with quadrupole excitation are [11] 
Table 1. Operating voltages

\begin{tabular}{ll}
\hline Sprayer & +3500 \\
Curtain plate & +500 \\
Orifice & $+50 ;+30$ \\
Q0 rod offset & +2 \\
Entrance lens & $+1,0,-1,-2$, or +120 \\
Q1 rod offset & $0,-1,-2,-3,-4,-5$ \\
Exit lens 1 & -15 or +120 \\
Exit lens 2 & -300 \\
\hline
\end{tabular}

$$
\omega_{e x}=\left|l+\beta_{u}\right| \frac{\Omega}{K}
$$

where $K=1,2,3, \ldots$ and $l=0, \pm 1, \pm 2 \ldots$ The $l=0$, $K=1$ resonances were excited.

Both dipole-dipole excitation and quadrupole excitation excite ions simultaneously in the $x$ and $y$ directions. With dipole-dipole excitation, the excitation field strength does not vary with position. With quadrupole excitation, the excitation field strength varies from zero at the quadrupole center to a maximum at the rods. All excitation voltages, dipole or quadrupole, are reported as $\mathrm{V}_{p-p}$ pole to ground.

For all the experiments with trapped ions reported here, $\sim 23 \%$ of the drive rf (in phase with the pair of poles that has the auxiliary excitation) was added to the exit lens by connecting the lens to the rf output of the quadrupole supply through a $15 \mathrm{pf}$ capacitor [12]. The quadrupole power supply (API3; MDS SCIEX, Concord, ON, Canada) was modified so that relatively low levels $(< \pm 15 \mathrm{~V})$ of resolving dc voltage could be added between the rod pairs. Operating voltages are shown in Table 1.

A schematic of the timing parameters for a typical axial ejection scan with trapped ions is shown in Figure $1 \mathrm{~b}$. The master clock is provided by a twochannel arbitrary waveform generator, (PC Instruments AWG-344; Twinsburg, OH). Each channel provides a maximum amplitude output (0 to peak) of 12 $\mathrm{V}$, which is increased to the desired voltage by homemade amplifiers. First a variable length (10-50 $\mathrm{ms}$ ) pulse, $1 \mathrm{~V}$ higher than the Q1 rod offset but below the Q0 rod offset $(+2 \mathrm{~V})$, is applied to the entrance lens to allow ions to enter Q1, (Q1 offset -1 to $-5 \mathrm{~V})$. During this injection step the exit lens is held at +120 $\mathrm{V}$. At the end of the injection pulse the entrance lens is raised to $+120 \mathrm{~V}$. Next, the trapped ions are cooled for $50 \mathrm{~ms}$. Just before the mass scan, the dc voltage applied to the exit lens is reduced to a scan value. For lower scan speeds this was 2 to $3 \mathrm{~V}$ above the dc rod offset of Q1 and for higher scan speeds, 10 to $25 \mathrm{~V}$ above the Q1 rod offset. Auxiliary dipole or quadrupole excitation is applied at a fixed frequency, and the drive rf is ramped over the chosen mass-to-charge range to eject ions from the linear trap. Finally, the dc voltages applied to the entrance lens and the exit lens are lowered to $-15 \mathrm{~V}$ for 10 to $50 \mathrm{~ms}$ to drain any residual ions from the linear ion trap and Q0.

To measure the number of ions injected into the trap, ions were injected (at $q=0.4$ ) as described above. After the cooling period, the exit lens voltage was lowered to $-15 \mathrm{~V}$ while the entrance was held at $+120 \mathrm{~V}$ and ions drained from the trap and were counted for $100 \mathrm{~ms}$ to give the number of injected ions. The ratio of the number of axially ejected ions to the number of injected ions gives the ejection efficiency.

Experiments were done with a conventional roundrod quadrupole rod array with a field radius $r_{0}=4.17$ $\mathrm{mm}$ and a ratio of rod radius to field radius $R / r_{0}=$ 1.126 , and with quadrupoles with $2.0 \%$ or $2.6 \%$ added octopole fields with field radii $\mathrm{r}_{0}=4.5 \mathrm{~mm}$. The octopole fields are added by making the radius of the $y$ rods, $R_{\mathrm{y}}$, greater than the radius of the $x$ rods, $R_{\mathrm{x}}\left(R_{\mathrm{x}}=\right.$ $\left.r_{0}\right)$. The quadrupole with a $2.0 \%$ added octopole field (i.e., $A_{4} / A_{2}=0.020$ ), had $R_{y} / R_{x}=1.220$ and the quadrupole with a $2.6 \%$ octopole field, $R_{y} / R_{x}=1.300$ [7]. The electric potential within these rod sets is given to a good approximation by

$$
\begin{aligned}
\Phi(x, y, t)= & \left(A_{0}+A_{2} \frac{x^{2}-y^{2}}{r_{0}^{2}}\right. \\
& \left.+A_{4} \frac{x^{4}-6 x^{2} y^{2}+y^{4}}{r_{0}^{4}}\right)\left(U-V_{r f} \cos \Omega t\right)
\end{aligned}
$$

where $x$ is the distance from the centre towards a smaller rod, $y$ is the distance from the center towards a larger rod, $U$ and $\mathrm{V}_{r f}$ are the amplitudes of $\mathrm{dc}$ and $\mathrm{rf}$ voltages applied from pole to ground, $A_{0}, A_{2}$, and $A_{4}$ are the dimensionless amplitudes of a constant potential, the quadrupole potential and the octopole potential respectively. For these rod sets $\mathrm{A}_{2} \approx 1.00$ and $A_{0} \approx-A_{4}$. Use of round rods adds other multipoles to the potential $[7,9]$.

Reserpine (m.w. 609.7) and renin substrate tetradecapeptide (m.w. 1759.0), were from Sigma (St. Louis, MO). Methanol and acetic acid were from Fisher Scientific (Nepean, ON, Canada). UHP grade nitrogen was from Praxair (Vancouver, BC, Canada). Solutions were as follows: reserpine, $1-10 \mu \mathrm{M}$, renin substrate $10-50$ $\mu \mathrm{M}$, both in $70 \%$ methanol, $29.5 \%$ water, $0.5 \%$ acetic acid. The solution flow rate for all experiments was 1 $\mu \mathrm{L} / \mathrm{min}$.

\section{Data Acquisition}

Different modes of applied rf, excitation and applied dc are possible and are summarized in Table 2. In principle, any of the three rf modes can be combined with any

\begin{tabular}{|c|c|c|}
\hline Drive RF & Excitation mode & DC between rods \\
\hline Balanced & None & None \\
\hline Unbalanced & $\begin{array}{l}\text { Dipole (smaller or } \\
\text { larger rods) }\end{array}$ & + Smaller rods \\
\hline rf on the exit lens & $\begin{array}{l}\text { Dipole-dipole } \\
\text { Quadrupole }\end{array}$ & + Larger rods \\
\hline
\end{tabular}
of the excitation modes and with any of the methods of

Table 2. Modes of applied rf, excitation, and dc 
applying dc (36 combinations). Each can be used with ions flowing continuously through the quadrupole or with trapped ions.

Spectra were acquired using a multichannel scalar (MCS) (MCS-pci; Ortec-Ametek, Oak Ridge, TN). The MCS is plugged into the pci-bus of an Intel Pentium III computer. The ramp output option of the MCS is used to scan the rf voltage of the quadrupole power supply. The ramp output can increase or decrease with time to give forward or reverse scans (i.e., ions injected in order of increasing or decreasing mass, respectively). A triangular ramp is used to initiate the start, end and final voltages. The start and end voltages determine the initial and final masses, and the final rf voltage is used for injection and draining of ions. There is electronic noise of about $8 \mathrm{mV}_{p-p}$ on the MCS ramp output, independent of the ramp output voltage. To minimize any effects of the noise on mass resolution the ramp output voltages were increased to 3-6 times greater than needed, and then reduced by resistive dividers to the required values. The noise was reduced by the same factor (i.e., 3-6).

Data analysis used in-house developed software. The software is designed to read MCS produced spectra with the corresponding parameters (e.g., rf voltage specifications) and save spectra in a database for later analysis and graphing. In a few steps a user can graph spectra as intensity versus mass-to-charge ratio, find peaks, and calculate resolution. The software is capable of storing and sorting thousands of spectra based on their collection dates. Using this software it was possible to organize and quickly browse data from a large number of experiments.

\section{Mass Calibration}

The rf and dc levels of the voltages applied to the quadrupole rods with conventional analysis are set by and are linearly proportional to a mass command voltage between 0 and $10.0 \mathrm{~V}$. To mass calibrate the spectra shown here, the quadrupole was first run conventionally with $\mathrm{rf} / \mathrm{dc}$ and calibrated so that the monoisotopic reserpine peak appeared at $m / z$ 609.3. In this case the rf level corresponds to the tip of the stability diagram where $q=0.70600$ [13]. Ions were then mass selectively ejected at various $q$ values, and the voltage at which they were ejected was determined from the MCS ramp parameters. The apparent mass was calculated from apparent mass $=($ ejection voltage $) * 609.3 /$ (voltage with conventional analysis).

The apparent mass did not correspond exactly to that expected from the $q$ value selected for ejection (see below). For the spectra of Figures 2, 3, 4, 5, and 6 , the masses were recalculated so that the monoisotopic peak appeared at the correct mass (e.g., 609.3 for reserpine).
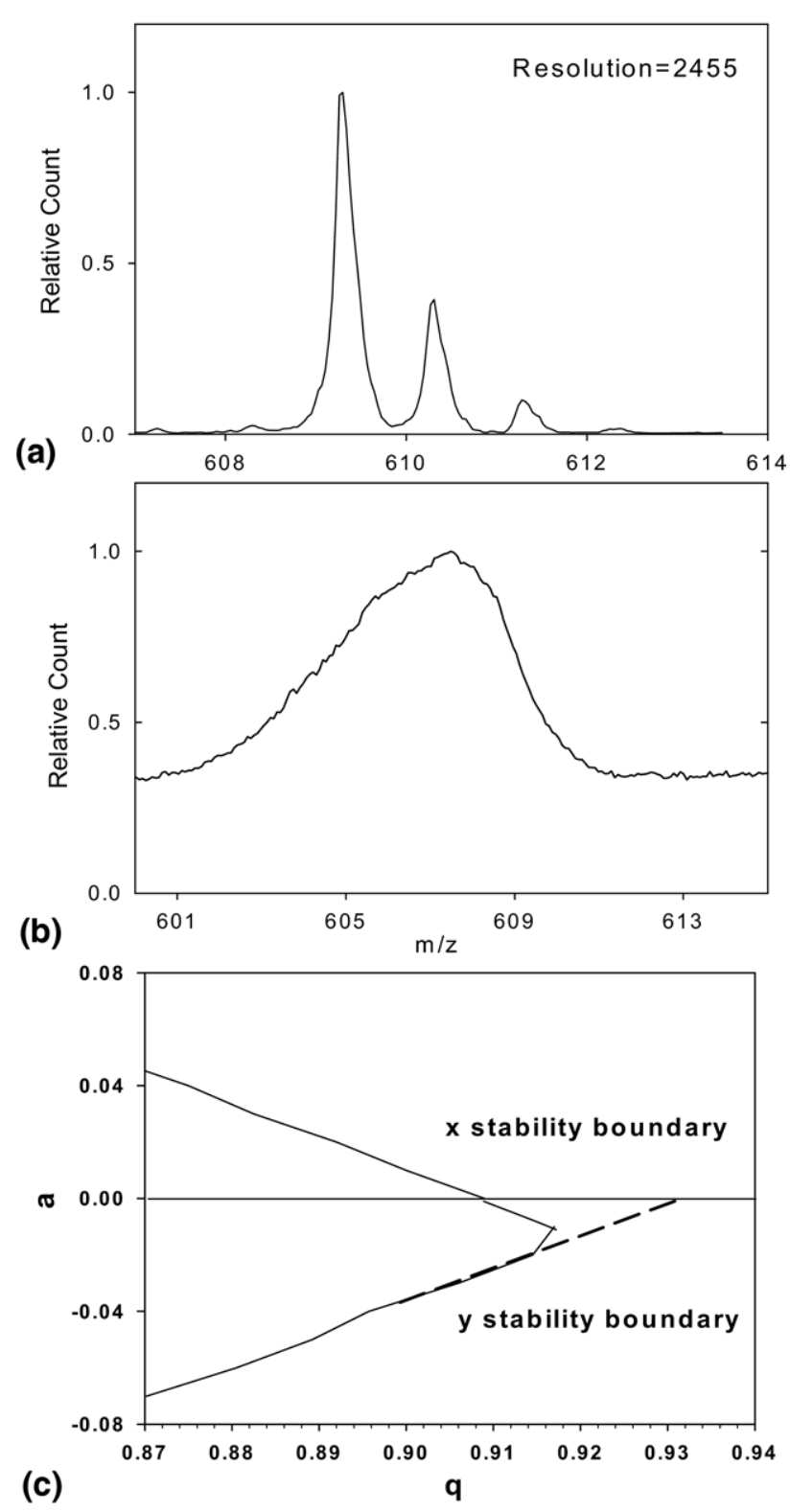

Figure 2. Experiments without trapping. (a) Axial ejection at $q=$ 0.908 with a conventional quadrupole. (b) Axial ejection near $q=$ 0.908 with a quadrupole with a $2.6 \%$ added octopole field. (c) Calculated stability boundaries of a quadrupole with a $2.6 \%$ added octopole field.

\section{Stability Diagram}

The stability boundaries for a quadrupole with a $2.6 \%$ added octopole field were calculated as in [13]. The transmission of the quadrupole for given $a, q$ values was calculated from 6000 ion trajectories. The initial spatial distributions were Gaussian with standard deviations $\sigma_{x}=\sigma_{y}=0.0020 r_{0}$. Initial velocity distributions were thermal for an ion of mass 390. Multipoles up to the 20 pole term were included in the calculation. No fringing fields were included. Points with a transmission of $1 \%$ were taken as the stability boundaries. 


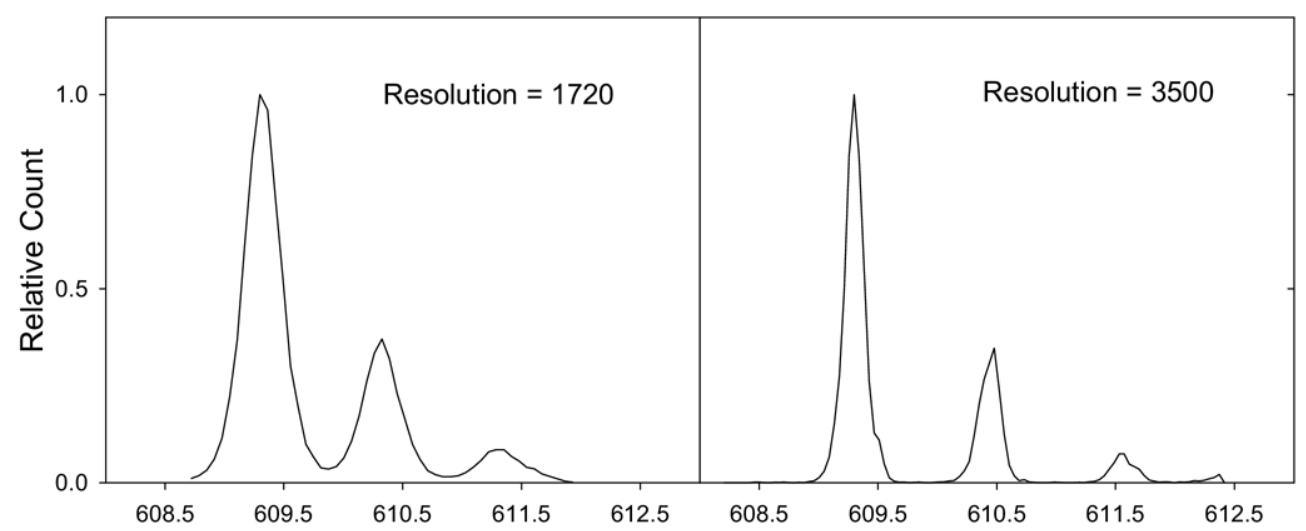

(a)

(b)

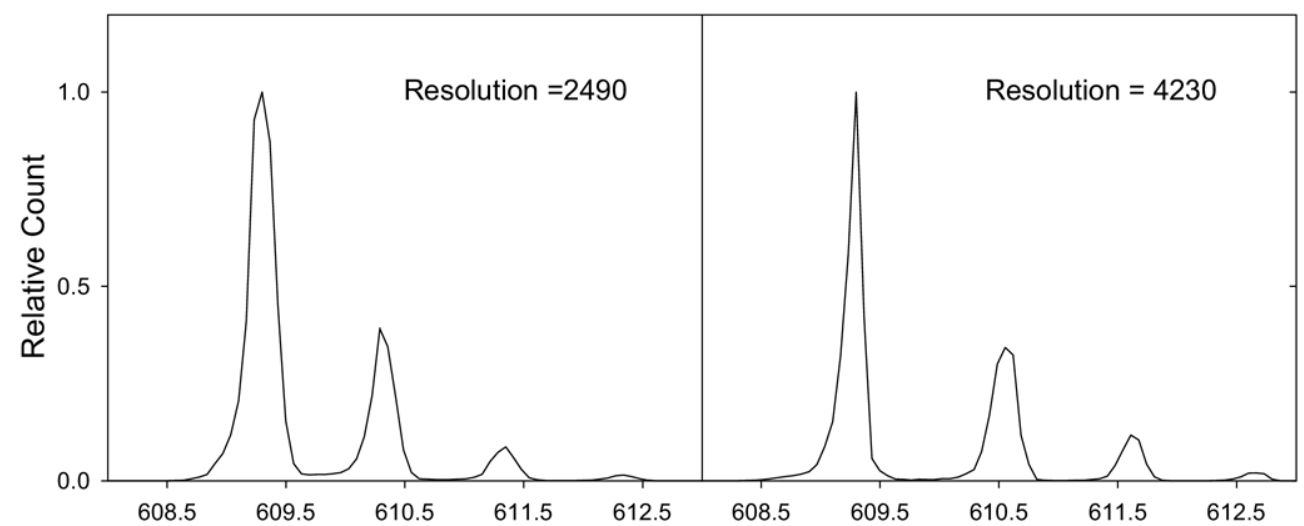

(c)

(d)

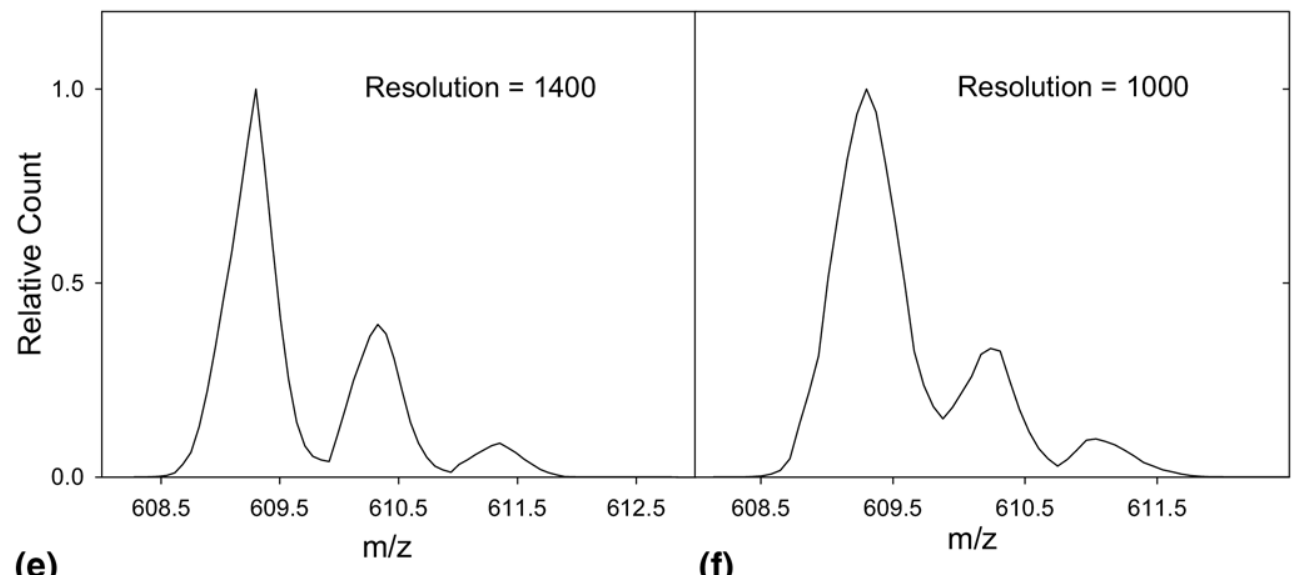

(e)

(f)

Figure 3. Peak shapes and resolution with axial ejection of trapped ions, and dipole excitation between the larger (y) rods. (a) Reverse scan, ejection at $q=0.40,10 \mathrm{Th} / \mathrm{s}$; (b) reverse scan, ejection at $q=0.80,10 \mathrm{Th} / \mathrm{s} ;(\mathbf{c})$ forward scan, ejection at $q=0.80,15 \mathrm{Th} / \mathrm{s} ;(\mathbf{d})$ reverse scan, ejection at $q=$ $0.80,15 \mathrm{Th} / \mathrm{s} ;(\mathbf{e})$ reverse scan, ejection at $q=0.80,1570 \mathrm{Th} / \mathrm{s} ;(\mathbf{f})$ reverse scan, ejection at $q=0.80,5000$ $\mathrm{Th} / \mathrm{s}$.

\section{Results and Discussion}

\section{Experiments Without Trapping}

In first experiments, ions flowed without trapping through Q1, operated in rf only mode. The amplitude of the drive rf was increased to bring ions of different $\mathrm{m} / \mathrm{z}$ ratios to the stability boundary $(a=0, q=0.908)$. Ions near the boundary gain large amplitudes of oscillation.
These excited ions overcome the exit lens barrier and are ejected to produce a mass spectrum [3].

Initially, experiments were done with a conventional quadrupole rod set using balanced and unbalanced (up to $15 \%$ ) rf, with $7 \mathrm{~V}$ dc applied between the rods and with 50 to $70 \mathrm{~V}_{p-p}$ auxiliary rf (at the frequency of quadrupole power supply) applied to the exit lens. This rf was $1.8 \%$ to $2.3 \%$ of the main rf and was in phase and 


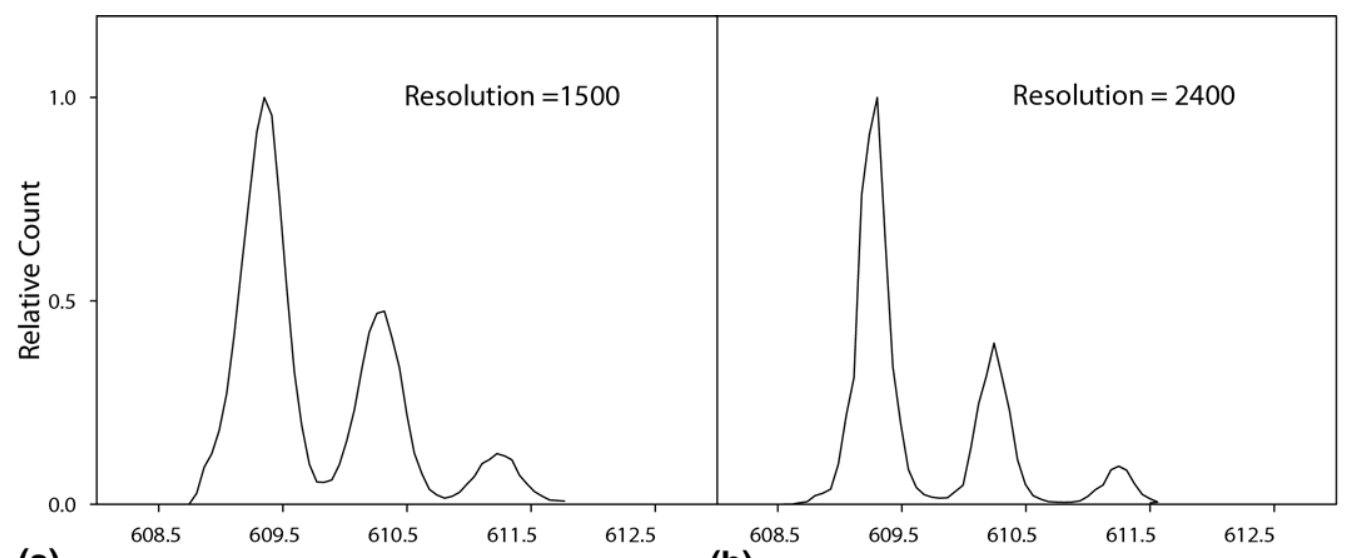

(a)

(b)

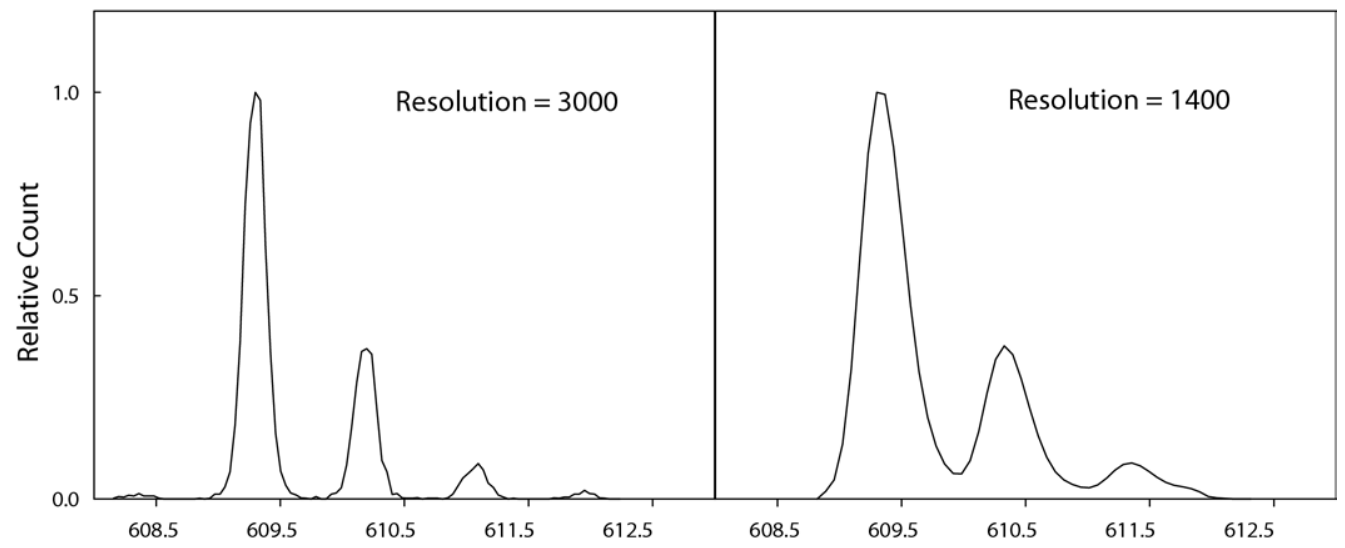

(c)

(d)

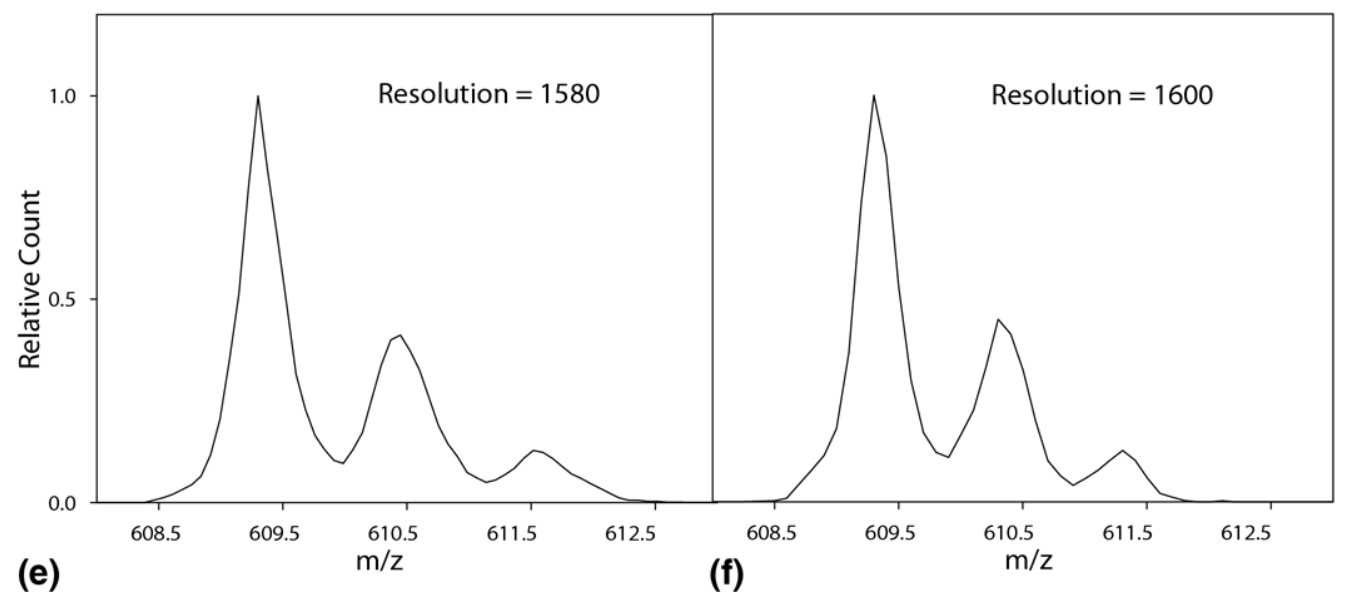

Figure 4. Peak shapes and resolution with axial ejection and dipole excitation between the smaller (x) rods. (a) Forward scan, ejection at $q=0.4,10 \mathrm{Th} / \mathrm{s}$; (b) forward scan, ejection at $q=0.80,15 \mathrm{Th} / \mathrm{s}$; (c) forward scan, ejection at $q=0.80,15 \mathrm{Th} / \mathrm{s}$; (d) reverse scan, ejection at $q=0.80,15 \mathrm{Th} / \mathrm{s}$; (e) forward scan, ejection at $q=0.80,1500 \mathrm{Th} / \mathrm{s}$; (f) forward scan, ejection at $q=0.80,5000 \mathrm{Th} / \mathrm{s}$.

phase locked with the drive rf. Experiments without this applied rf gave similar results. These experiments gave results similar to those of Hager [3]. Figure 2a shows a spectrum of the isotopic peaks of reserpine, obtained with $\pm 15 \%$ unbalanced $\mathrm{rf}$ and ejection at the stability boundary $q=0.908$ with a scan speed of 0.8 $\mathrm{Th} / \mathrm{s}$. The resolution possible in axial ejection depends on the scan speed. In this and other experiments, low scan speeds of ca. 1-15 Th/s were used to allow comparison with Hager's experiments [3, 4]. For many applications, these low scan speeds would only be of use for scans over very limited mass ranges.

Similar axial ejection experiments without trapping were then tried with a quadrupole with a $2.6 \%$ added octopole field. With balanced $\mathrm{rf}$ and no dc between the rods, and a scan speed of $3 \mathrm{Th} / \mathrm{s}$, a very broad peak 


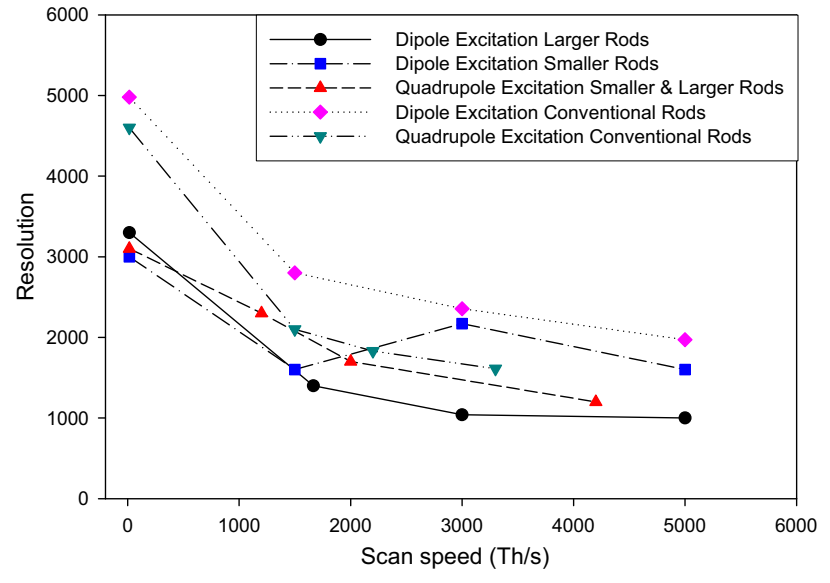

Figure 5. Resolution versus scan speed with different excitation modes, with a conventional quadrupole and a quadrupole with a $2.0 \%$ added octopole field.

with poor resolution (nominal $\mathrm{R}_{1 / 2}=76$ ) was produced at about $\mathrm{m} / \mathrm{z}=607$, Figure $2 \mathrm{~b}$. Combinations of unbalanced $\mathrm{rf}$, dc between the rod pairs, and rf on the exit lens were tried, but the resolution could not be improved. Evidently with axial ejection at $q=0.908$ and with excitation at the stability boundary, the quadrupole with $2.6 \%$ added octopole field produces much lower resolution than a conventional quadrupole.

The lower resolution may be a result of changes to the stability boundaries caused by the octopole field. With a conventional rod set, at the $a=0, q=0.908$ boundary, ion motion becomes unstable in the $x$ and $y$ directions simultaneously. The stability boundaries of a quadrupole with $2.6 \%$ octopole field are shown in Figure 2c. The $x$ and $y$ motions have separate stability boundaries on the $a=0$ axis. The $x$ boundary is at $q=$ 0.908 . Extrapolation of the y stability boundary to $a=0$ (the dashed line in Figure 2c) shows the boundary is at about $q=0.931$. The $y$ boundary is diffuse [9]. The octopole field couples the $x$ and $y$ motions. Excitation of ions in the $x$ direction near $q=0.908$ may lead to excitation in the $y$ direction where motion is stable, resulting in delayed ejection and low-resolution.

\section{Experiments with Trapped Ions}

Dipole excitation with a quadrupole with a $2.0 \%$ added octopole field. Initial experiments with trapped ions and a quadrupole with a $2.0 \%$ added octopole field were tried with ejection at the stability boundary near $q=0.908$. As with experiments without trapping, only very low resolution was found. Therefore all subsequent experiments with trapped ions used auxiliary ac voltages to excite ions for axial ejection.

Axial ejection experiments with dipole excitation between the smaller or larger rods were done with a quadrupole with a $2.0 \%$ added octopole field. When the quadrupole is used as Q3 in a triple quadrupole system [4], it is necessary to operate the rod set as both a conventional rf/dc mass analyzer and a linear ion trap with axial ejection. Operation as a mass filter requires that the positive dc be applied to the smaller rods [9]. Experiments by Hager [3] showed that in some cases the ion signal with axial ejection could be increased by using a combination of unbalanced rf applied to the quadrupole and low dc voltages applied between the rod pairs, to modify the exit fringing field. An unbalanced $\mathrm{rf}$ is equivalent to a balanced $\mathrm{rf}$ with an added axis potential between the quadrupole and exit lens [14]. This potential difference between the quadrupole and exit lens can be produced by applying some of the quadrupole rf to the exit lens without the need to

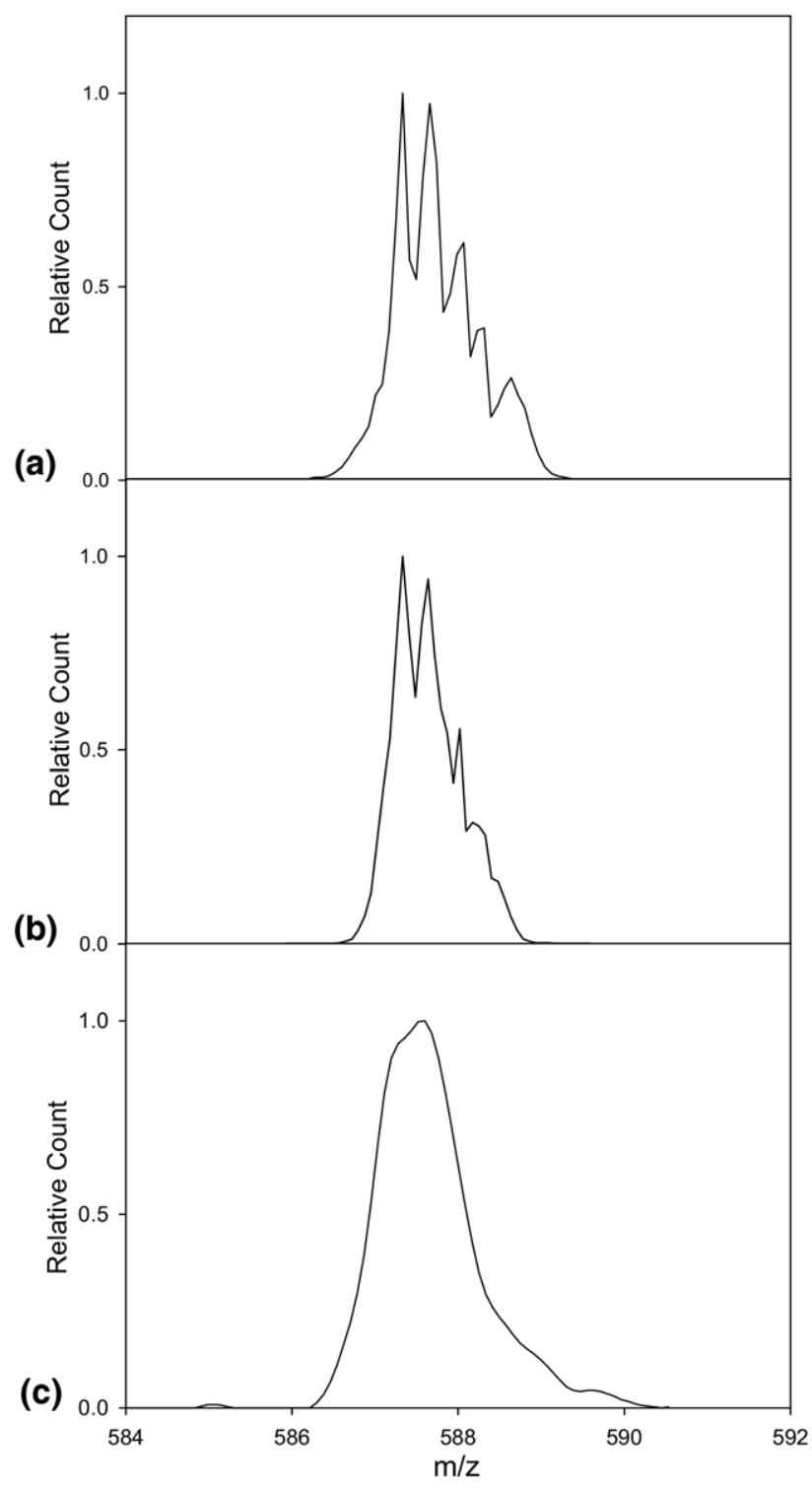

Figure 6. Axial ejection of +3 ions of renin substrate tetradecapeptide with dipole excitation applied to (a) conventional rods with a forward scan and ejection at $q=0.80,(\mathbf{b})$ the smaller rods of a quadrupole with a $2.0 \%$ octopole field with a forward scan and ejection at $q=0.80$, and (c) the larger rods of a quadrupole with a $2.0 \%$ octopole field with a reverse scan and ejection at $q=$ 0.20 . The scan speed in all cases is $3000 \mathrm{Th} / \mathrm{s}$. 
unbalance the rf. In later work, Hager and Londry showed that applying some of the trapping $\mathrm{rf}$ to the exit lens can improve the resolution, with no loss of ion signals [12]. Thus, in all experiments with trapped ions, the drive rf was balanced and the positive dc was applied to the smaller rods [9]. The polarity of the dc applied between the rods was reversed and it was found that this gave no improvements in resolution. With low scan speeds (10-15 Th/s), no resolving dc was added to the rods. Adding dc did not improve the resolution. With high scan speeds (>300 Th/s), adding the low and optimum levels of dc that are indicated below helped to improve the ejection efficiency and resolution.

Initially, ejection with excitation frequencies corresponding to $q$ values of $0.4,0.5,0.6,0.7$, and 0.8 were tested. Figure $3 \mathrm{a}, \mathrm{b}$ and Figure $4 \mathrm{a}, \mathrm{b}$ show results of ejection at $q=0.4$ and $q=0.8$, respectively, with excitation between the larger rods (reverse scans) and smaller rods (forward scans) respectively. For these figures the exit barrier (the exit lens voltage minus the Q1 rod offset voltage) was $1.0 \mathrm{~V}$. No resolving dc was added. Higher $q$ values result in higher resolution. With dipole excitation applied to the larger rods and reverse scanning, excitation amplitudes of 0.03 to $0.1 \mathrm{~V}_{p-p}$ at scan speeds of 10 to $15 \mathrm{Th} / \mathrm{s}$ gave the best peak shape and resolution. With dipole excitation applied to the smaller rods and forward scanning, an excitation amplitude of $0.30 \mathrm{~V}_{p-p}$ at scan speeds of 10 to $15 \mathrm{Th} / \mathrm{s}$, was optimal.

Two factors may contribute to the higher resolution at higher $q$ values. First, ions have higher oscillation frequencies at higher $q$. If there is fixed range of ejection frequencies caused by say damping, the frequency resolution $f / \Delta f$ and hence mass resolution, $m / \Delta m$ increase with $q$ [15]. Second, $\beta$ and hence, the resonant frequency of an ion, is a nonlinear function of $q$ and increases more rapidly with $q$ at higher $q$ values. This increases the rate of change of frequency with mass,

$$
\left(\frac{d f}{d m}=\frac{d f}{d q} \frac{d q}{d m}=-\frac{q}{m} \frac{d f}{d q}\right)
$$

which may also increase mass resolution.

Because ejection at higher $q$ gives higher resolution, subsequent experiments used ejection at $q=0.80$ (413.14 kHz auxiliary ac). Figure $3 \mathrm{c}$ and d show a comparison between forward and reverse scans with excitation between the larger rods (15 Th/s, exit barrier $5.0 \mathrm{~V})$. No dc between the rods was added. Higher resolution is possible with reverse scans. While Figure $3 \mathrm{~b}$ and $\mathrm{d}$ both show reverse scans at with ejection at $q=$ 0.80 , Figure $3 \mathrm{~d}$ shows somewhat higher resolution than Figure $3 b$ because of the higher exit barrier used for Figure 3d. Figure $4 c$ and $d$ compare forward and reverse scans with excitation between the smaller rods (15 Th/s, exit barrier $4.7 \mathrm{~V}$ ). No dc between the rods was added. In this case forward scans give higher resolution. Figure 4c shows somewhat higher resolution than Figure $4 \mathrm{~b}$ because of the higher exit barrier. These results are as expected from the frequency shifts caused by the added octopole field [16]. The shift of oscillation frequency, $\Delta \omega$, in a quadrupole with an added octopole is [8]

$$
\Delta \omega=3 \frac{A_{4}}{A_{2}}\left(\frac{b^{2}}{r_{0}^{2}}\right) \omega_{0}
$$

where $b$ is the amplitude of oscillation, and $\omega_{0}$ is the resonant frequency of ions in the quadrupole without an octopole field. With $\mathrm{A}_{4}>0$ and with excitation between the smaller rods ( $x$ motion) there is a shift to higher frequency with increasing amplitude. For the $y$ motion there is a shift to lower frequency of the same magnitude for the same amplitude of oscillation. With excitation between the smaller rods and forward scanning, as the rf amplitude increases, the oscillation frequencies of the ions increase. As the ions come into resonance their frequencies are further increased by the octopole component. These frequency shifts drive the ions faster toward the frequency of the auxiliary ac, which in turn causes the ion oscillation amplitudes to grow more rapidly until ions are ejected from the trap. With excitation between the smaller rods and reverse scanning, as the rf amplitude decreases the oscillation frequencies of the ions decrease. As the ions come into resonance their frequencies are increased by the octopole component. These frequency shifts cause the ions to move away from the frequency of the auxiliary ac, which in turn causes the oscillation amplitudes to grow more slowly, and this degrades mass resolution. For similar reasons, reverse scans give higher resolution with excitation between the larger rods.

The apparent masses at which ions of $m / z 609.3$ are ejected are consistent with this explanation and are shown in Table 3. (The resolutions for these scans are as shown in Figures 3 and 4.) With forward scans, and excitation between the smaller rods, ions are ejected at an apparent mass of 685.56. With the ejection between the larger rods, the ions are ejected at 686.15, showing that ejection is delayed and the quadrupole must scan up to a somewhat higher rf voltage before the ions are ejected. With a reverse scan and excitation between the larger rods, ions are ejected 689.32. With excitation between the smaller rods, the ions are ejected at 688.53, showing again that ejection is delayed and the quadru-

Table 3. Apparent mass of reserpine $m / z 609.28$ with different scan directions with trapped ions excited between the smaller and larger rods at $q \approx 0.80$ and a scan speed of $10 \mathrm{Th} / \mathrm{s}$

\begin{tabular}{ccc}
\hline Scan direction & $\begin{array}{c}\text { Rod pair with } \\
\text { applied excitation }\end{array}$ & $\begin{array}{c}\text { Apparent mass } \\
\text { of } \mathrm{m} / \mathrm{z} 609.28\end{array}$ \\
\hline \hline Forward & Smaller & 685.56 \\
Forward & Larger & 686.15 \\
Reverse & Larger & 689.32 \\
Reverse & Smaller & 688.53 \\
\hline
\end{tabular}


pole must scan down to a lower rf voltage before the ions are ejected.

The effects of higher scan speeds were investigated. Figure $3 e$ and $f$ show spectra of reserpine ions with excitation between the larger rods and scan speeds of 1570 and $5000 \mathrm{Th} / \mathrm{s}$, respectively (reverse scans, exit barrier $9.5 \mathrm{~V}$ ). There was $10 \mathrm{~V}$ dc added between the rods. At higher scan speeds, with dipole excitation applied to the larger rods and reverse scanning, an excitation amplitude of 0.55 to $1.0 \mathrm{~V}_{p-p}$ at scan speeds of 3000 to $5000 \mathrm{Th} / \mathrm{s}$ gave the best peak shape and resolution. Figure $4 \mathrm{e}$ and $\mathrm{f}$ show spectra of reserpine ions with excitation between the smaller rods at scan speeds of $1500 \mathrm{Th} / \mathrm{s}$ (with $7 \mathrm{~V} \mathrm{dc}$ ) and $5000 \mathrm{Th} / \mathrm{s}$ (with $10 \mathrm{~V} \mathrm{dc}$ ), respectively (forward scans, exit barrier 20.0 V). With dipole excitation applied to the smaller rods and forward scanning, an excitation amplitude of 10 $\mathrm{V}_{p-p}$ at scan speeds of 3000 to $5000 \mathrm{Th} / \mathrm{s}$ was optimal. At scan speeds of ca. $1500 \mathrm{Th} / \mathrm{s}$ (Figures 3e and 4e) the resolution is about 1500 . At $5000 \mathrm{Th} / \mathrm{s}$ (Figures $3 \mathrm{f}$ and 4f) the peak widths and spacing are not constant, particularly with reverse scans, Figure 3f. To calculate the resolution in this case, the peak width of the most abundant peak (the monoisotopic peak) was compared with the spacing between the two most abundant isotopic peaks, taken as one mass unit. Resolutions of ca. 1000 (Figure 3f) and 1600 (Figure 4f) are calculated. The unequal peak widths and spacings are likely a result of a limitation of the quadrupole power supply, which was designed for scan speeds of ca. $100 \mathrm{Th} / \mathrm{s}$ with step sizes of $0.1 \mathrm{Th}$.

Dipole-dipole excitation with a quadrupole with a $2.0 \%$ added octopole field. Experiments were tried with dipole excitation applied simultaneously between the smaller and larger rods with no resolving dc added to the rods, and with scan speeds of $10 \mathrm{Th} / \mathrm{s}$. When the same excitation frequency and amplitude $(413.14 \mathrm{kHz}$ and 0.2 $\mathrm{V}_{p-p}$ ) were applied to both rod pairs, with forward and reverse scanning the ions were ejected mainly at the apparent masses of $\mathrm{m} / \mathrm{z}=686.15$ and $\mathrm{m} / \mathrm{z}=689.32$, respectively. These are the apparent masses at which ions are ejected with excitation between the larger rods alone. Approximately $10 \%$ of the ejected ions appeared at the apparent masses corresponding to excitation between the smaller rods, $\mathrm{m} / \mathrm{z}=685.56$ and $\mathrm{m} / \mathrm{z}=$ 688.53 for forward and reverse scans, respectively. The excitation frequency applied to the smaller rods was then increased to $413.9 \mathrm{kHz}$, so that ions were ejected at the apparent masses corresponding to excitation between the larger rods. With reverse scanning and a scan speed of $10 \mathrm{Th} / \mathrm{s}$, a resolution of 2000 was possible. This gave resolution lower than that with dipole excitation between the larger rods alone (Figure $3 b$ ). With forward scanning the resolution decreased to 1200 . Thus, no improvements were obtained by applying dipole excitation simultaneously between both rod pairs and no additional experiments with faster scans were done.
Quadrupole excitation with a quadrupole with a $2.0 \%$ added octopole field. Auxiliary quadrupole excitation was applied to the quadrupole rods with a $2.0 \%$ added octopole field to give parametric excitation at twice the fundamental frequency of ion oscillation $(l=0, K=1$ in eq 2). Due to an electronics limitation, the higher auxiliary voltages at the higher frequencies needed for quadrupole excitation at $q=0.80$ could not be applied, so experiments were performed with ions trapped at $q=0.7002$ (excitation frequency $676.00 \mathrm{kHz}$ ). The optimal excitation amplitude for low scan speeds (10-15 $\mathrm{Th} / \mathrm{s}$ ) was $1.14 \mathrm{~V}_{p-p}$ and the highest possible excitation amplitude for higher scan speeds (3000-5000 Th/s) was $3.64 \mathrm{~V}_{p-p}$.

With forward scans at $15 \mathrm{Th} / \mathrm{s}$, a resolution of about 1800 was obtained. With reverse scans at $15 \mathrm{Th} / \mathrm{s}$ a resolution of about 3100 was possible. In both cases no resolving dc was added to the rods. These resolutions are similar to those obtained with dipole excitation between the larger rods. Quadrupole excitation excites ion motion between both the smaller and larger rods. It appears as if the motion between the larger rods dominates the ejection process. Most experiments with quadrupole excitation investigated reverse scanning because this yields higher resolution. With reverse scans at $1200 \mathrm{Th} / \mathrm{s}$ and $7.0 \mathrm{~V}$ dc between the rods, a resolution of 2300 was obtained, and at $4200 \mathrm{Th} / \mathrm{s}$, a resolution of 1200 was achieved. Overall no advantage to using quadrupole excitation was found.

\section{Experiments with a Conventional Quadrupole Rod Set}

Dipole excitation was used to excite ions in a conventional rod set at $q=0.80$ (excitation frequency 413.14 $\mathrm{kHz}$, amplitude $\left.0.03 \mathrm{~V}_{p-p}\right)$. For the experiments described here for scan speeds of $15 \mathrm{Th} / \mathrm{s}$, there was no resolving dc added to the rods. For all the other reported scan speeds, there was $7 \mathrm{~V}$ dc added between the rods. With dipole excitation applied to either pair of rods, forward and reverse scans at low scan speed (15 Th/s) gave similar spectra, with resolutions of up to 4600. With forward scans, at $3300 \mathrm{Th} / \mathrm{s}$ (excitation amplitude $1.0 \mathrm{~V}_{p-p}$ ), the resolution was 2350 . With forward scans at $5000 \mathrm{Th} / \mathrm{s}$ (excitation amplitude 2.0 $\left.\mathrm{V}_{p-p}\right)$, peaks showed mass shifts and unequal spacings, which, as stated, were probably due to a limitation of our quadrupole power supply. The resolution, calculated as described above, was 1970. With reverse scans at $5000 \mathrm{Th} / \mathrm{s}$ a similar resolution, 2000 (same method of calculation), was achieved.

With quadrupole excitation $(l=0$ and $K=1$ in eq 2, $\left.q=0.7002,676.00 \mathrm{kHz}, 0.90 \mathrm{~V}_{p-p}\right)$, and with a scan speed of $15 \mathrm{Th} / \mathrm{s}$, both forward and reverse scans gave a resolution of 4300 to 4700 . High scan speed was also possible with both forward and reverse scanning. With $7 \mathrm{~V} \mathrm{dc}$ added between the rods, forward scanning (3.76 $\left.\mathrm{V}_{p-p}\right)$ and a scan speed of $3800 \mathrm{Th} / \mathrm{s}$, the resolution was 
1580 , and with reverse scanning $\left(3.65 \mathrm{~V}_{p-p}\right)$ and a scan speed of $3300 \mathrm{Th} / \mathrm{s}$, resolution of 1500 was possible. Scan speeds higher than 3300 to $3800 \mathrm{Th} / \mathrm{s}$ in each scanning direction resulted in poor peak shapes. Thus, as with the quadrupole with added octopole field, no advantage was found with using quadrupole excitation.

Experiments were done with dipole excitation applied simultaneously to both pairs of rods. The optimal excitation amplitudes for different scan speeds ranged from $0.05 \mathrm{~V}_{p-p}$ at $15 \mathrm{Th} / \mathrm{s}$ to $3.5 \mathrm{~V}_{p-p}$ at $5000 \mathrm{Th} / \mathrm{s}$. With forward scans, low scan speeds (15 Th/s) gave a resolution of around 4600. With increasing scan speed, about $3000 \mathrm{Th} / \mathrm{s}$, a resolution of 2100 was possible, and with a scan speed at $5000 \mathrm{Th} / \mathrm{s}$, the resolution was 1500 . Dipole-dipole excitation resulted in lower resolution at higher scan speeds than single dipole excitation. Again, no improvements in scan speed or resolution were found with dipole-dipole excitation.

Figure 5 shows a comparison of scan speeds and resolutions achieved with each mode of excitation for a conventional quadrupole and a quadrupole with a $2.0 \%$ added octopole field. In general, increasing scan speed decreases resolution. The quadrupole with a $2.0 \%$ added octopole field shows somewhat lower resolution than a conventional rod set at a given scan speed. In all cases resolutions of 1000 or more can be obtained at scan speeds up to $5000 \mathrm{Th} / \mathrm{s}$.

\section{Optimum Operating Mode with a Quadrupole with an Added Octopole Field}

Mass selective axial ejection of trapped ions that have a broad range of mass to charge ratios, requires ejection at a high $q$ value with forward scans. In this case, dipole excitation between the smaller rods gives the highest resolution and is the preferred mode of operation. Conversely, with reverse scans, a broad range of mass to charge ratios can be mass analyzed with ejection at low $q$. (With reverse scans, ejection at high $q$ allows only a very limited mass range of trapped ions to be stored and ejected, for example $m / z$ 537-609 with ejection at $q=0.80$.) For reverse scans, excitation between the larger rods gives higher resolution and is preferred. However any gain in resolution from exciting between the larger rods is offset by ejecting at lower $q$. Forward scans with ejection at $q=0.80$ were compared to reverse scans with ejection at $q=0.20$, with the +3 ions of renin substrate tetradecapeptide with a conventional quadrupole rod set and a rod set with a $2.0 \%$ added octopole field. In all cases $10 \mathrm{~V}$ dc was added between the rods. Figure 6 a shows the resolution achieved with a conventional rod set with forward scanning and ejection at $q=$ 0.80 , at $3000 \mathrm{Th} / \mathrm{s}$ scan speed and a step size of 0.080 mass units. The resolution is just sufficient to separate the isotopic peaks. Figure $6 \mathrm{~b}$ shows the peak shape and resolution with the quadrupole with a $2.0 \%$ added octopole field, dipole excitation between the smaller rods, ejection at $q=0.80$, a forward scan at $3000 \mathrm{Th} / \mathrm{s}$, and step size 0.08 mass units. Although the resolution is slightly lower than with a conventional rod set, the isotopic peaks are (just) resolved. With dipole excitation between the larger rods, reverse scanning and ejection at $q=0.20$, at $3000 \mathrm{Th} / \mathrm{s}$ and a step size of 0.08 mass units, it was not possible to resolve the isotopic peaks, Figure 6c. Thus, of all the operating modes investigated here, dipole excitation between the smaller rods with ejection at high $q$ is preferred when ions of a broad range of $m / z$ are to be mass analyzed.

\section{Ejection Efficiencies}

With trapped ions, the operating conditions can be chosen to give the highest resolution or to give the highest sensitivity at each scan speed. Lowering the exit barrier increases sensitivity but decreases resolution. The ejection efficiencies of the conventional rod set and the rod set with a $2.0 \%$ added octopole field were measured with the results shown in Table 4 . Here the system was optimized for the highest resolution with dipole excitation to eject ions at $q=0.80$, with forward scans. The ejection efficiency decreases as the scan speed increases. The two rod sets show similar efficiencies. The system was then optimized for the highest ejection efficiency while keeping $R_{1 / 2} \approx 1000$ at each scan speed, and the ejection efficiencies with the quadrupole with a $2.0 \%$ added octopole field were remeasured, with the results shown in Table 4 . The ejection efficiencies here are higher, with the greatest increases at the higher scan speeds. Hager found efficiencies of $2 \%$ to $18 \%$ with ejection of ions from Q2 of a triple quadrupole system with operating pressures of ca. $1 \times$ $10^{-4}$ torr to $2 \times 10^{-3}$ torr [4]. Higher pressures gave higher efficiencies. Because of the much higher operating pressures in those experiments, the results cannot be compared directly with the experiments here.

Table 4. Ejection efficiencies (\%)

\begin{tabular}{|c|c|c|}
\hline Scan speed Th/s & $\begin{array}{l}\text { Conventional } \\
\text { quadrupole }\end{array}$ & $\begin{array}{l}\text { Quadrupole with } \\
\text { a } 2.0 \% \text { added } \\
\text { octopole field }\end{array}$ \\
\hline \multicolumn{3}{|c|}{ System optimized for the highest resolution } \\
\hline 15 & 30 & 60 \\
\hline 600 & $2.3^{\mathrm{a}}$ & 2.0 \\
\hline 1500 & 2.0 & 1.8 \\
\hline 3000 & 1.3 & 1.0 \\
\hline 5000 & 0.5 & 0.4 \\
\hline \multicolumn{3}{|c|}{ System optimized for the highest sensitivity } \\
\hline 15 & & 67 \\
\hline 267 & & 38 \\
\hline 671 & & 5.6 \\
\hline 1706 & & 3.7 \\
\hline 2542 & & 2.5 \\
\hline 3917 & & 2.4 \\
\hline
\end{tabular}

${ }^{a}$ Measured at $666 \mathrm{Th} / \mathrm{s}$. 


\section{Conclusions}

Comparison of mass selective axial ejection with a conventional quadrupole rod set and a quadrupole with a $2.0 \%$ added octopole field with different modes of excitation shows similar resolutions and ejection efficiencies, with somewhat higher resolution obtained with a conventional quadrupole rod set. Nevertheless, mass selective axial ejection with quadrupoles with added octopole fields is possible with a resolution of several thousand and scan speeds of at least $2000 \mathrm{Th} / \mathrm{s}$. Thus, quadrupoles with added octopole fields can be used as rf/dc mass filters, demonstrate higher efficiency in MS/MS experiments, and can eject ions axially in a mass selective manner with high resolution and scan speed. Quadrupoles with added octopole fields show promise as improved ion traps, particularly for instruments where Q3 of a triple quadrupole is used as a linear trap.

\section{Acknowledgments}

The authors acknowledge support by the Natural Sciences and Engineering Research Council and MDS-SCIEX through an Industrial Research Chair. They thank Zilan Xiao for calculating the stability boundaries of a quadrupole with a $2.6 \%$ added octopole field.

\section{References}

1. Church, D. A. Storage-Ring Ion Trap Derived from the Linear Quadrupole Radiofrequency Mass Filter. J. Appl. Phys. 1969, 40, 3127-3134.
2. Douglas, D. J.; Frank, A. J.; Mao, D. M. Linear Ion Traps in Mass Spectrometry. Mass Spectrom. Rev. 2004, 23, 1-29.

3. Hager, J. W. Performance Optimization and Fringing Field Modifications of a 24-mm Long RF-Only Quadrupole Mass Spectrometer. Rapid Commun. Mass Spectrom. 1999, 13, 740-748.

4. Hager, J. W. A New Linear Ion Trap Mass Spectrometer. Rapid Commun. Mass Spectrom. 2002, 16, 512-526.

5. Wang, Y.; Franzen, J.; Wanczek, K. P. The Nonlinear Resonance Ion Trap. 2. A General Theoretical Analysis. Int. J. Mass Spectrom. Ion Processes 1993, 124, 125-144.

6. Wang, Y.; Franzen, J. The Nonlinear Ion Trap. 3. Multipole Components in Three Types of Practical Ion Trap. Int. J. Mass Spectrom. Ion Processes 1994, 132, 155-172.

7. Sudakov, M.; Douglas, D. J. Linear Quadrupoles with Added Octopole Fields. Rapid Commun. Mass Spectrom. 2003, 17, 2290-2294.

8. Michaud, A. L.; Frank, A. J.; Ding, C.; Zhao, X. Z.; Douglas, D. J. Ion Excitation in a Linear Quadrupole Ion Trap with an Added Octopole Field. J. Am. Soc. Mass Spectrom. 2005, 16, 835-849.

9. Ding, C. F.; Konenkov, N. V.; Douglas, D. J. Quadrupole Mass Filters with Octopole Fields. Rapid Commun. Mass Spectrom. 2003, 17, 24952502.

10. Campbell, J. M.; Collings, B. A.; Douglas, D. J. A New Linear Ion Trap Time-of-Flight System with Tandem Mass Spectrometry Capabilities. Rapid Commun. Mass Spectrom. 1998, 12, 1463-1474.

11. Sudakov, M.; Konenkov, N.; Douglas, D. J.; Glebova, T. Excitation Frequencies of Ions Confined in a Quadrupole Field with Quadrupole Excitation. J. Am. Soc. Mass Spectrom. 2000, 11, 10-18.

12. Hager, J.W.; Londry, F. A. System and Method for Modifying the Fringing Field of a Radio Frequency Multipole; US Patent 7019290, 2006

13. Dawson, P. H. Principles of Operation. In Quadrupole Mass Spectrometry and Its Applications; Dawson, P. H., Ed.; AIP Press: Woodbury, N.Y., 1995, p 21.

14. Douglas, D. J.; Glebova, T. A.; Konenkov, N. V.; Sudakov, M. Y. Spatia Harmonics of the Field in a Quadrupole Mass Filter with Circular Electrodes. Tech. Phys. 1999, 44, 1215-1219.

15. Goeringer, D. E.; Whitten, W. B.; Ramsey, J. M.; McLuckey, S. A.; Glish G. L. Theory of High-Resolution Mass-Spectrometry Achieved Via Resonance Ejection in the Quadrupole Ion Trap. Anal. Chem. 1992, 64, 1434-1439.

16. Williams, J. D.; Cox, K. A.; Cooks, R. G.; McLuckey, S. A.; Hart, K. J. Goeringer, D. E. Resonance Ejection Ion-Trap Mass-Spectrometry and Nonlinear Field Contributions-the Effect of Scan Direction on Mass Resolution. Anal. Chem. 1994, 66, 725-729. 